\title{
Harmonic maps from the complex plane into surfaces with nonpositive curvature
}

\author{
Zheng-Chao Han, Luen-Fai TAM ${ }^{1}$ \\ ANDREJS TREIBERGS ${ }^{2}$ AND TOM WAN ${ }^{3}$
}

\begin{abstract}
We give a characterization for an orientation preserving harmonic diffeomorphism from $\mathbb{C}$ into a complete, simply connected, negatively pinched surface to have a polynomial growth Hopf differential. In particular, we prove that an orientation preserving harmonic diffeomorphism from $\mathbb{C}$ into the Poincaré disk $\mathbb{H}$ has a polynomial growth Hopf differential of degree $m$ if and only if its image is an ideal polygon with $m+2$ vertices on $\partial \mathbb{H}$, with the assumption that the conformal metric on $\mathbb{C}$ with the $\partial$-energy density as the conformal factor is complete. We will describe the geometric behavior of this harmonic diffeomorphisms in terms of the trajectories of their Hopf differentials. We will also construct all harmonic diffeomorphisms in this class, and prove that there is an $m-1$ parameter family of nontrivially distinct harmonic diffeomorphisms from the complex plane to a fixed ideal polygon with $m+2$ vertices in the hyperbolic plane. In particular, such harmonic maps are not unique, answering a question of Schoen.
\end{abstract}

\section{Introduction.}

This paper addresses some questions regarding the geometric behavior of harmonic maps between surfaces. Harmonic maps are closely related to the deformation theory of Riemann surfaces. One of the questions that arise naturally in the deformation theory is: whether Riemann surfaces which are related by a harmonic diffeomorphism are necessarily quasi-conformally related? The case of interest is of course non-compact surfaces. See R.

1991 Mathematics Subject Classification: Primary 58E20, Secondary 53C42, 53C50.

${ }^{1}$ Partially supported by NSF grant

${ }^{2}$ Partially supported by NSF grant

${ }^{3}$ Partially supported by the Earmarked Grant of Hong Kong and Summer Research Grant 1994 of the Chinese University of Hong Kong 
Schoen's article [S] for a general discussion of this area, where this and other questions were discussed. Note that a map between surfaces being harmonic depends only on the conformal structure of the domain surface, not on its metric structure, but depends on the metric of the target surface in an essential way. There is a theorem of Heinz [He] which says that there is no harmonic diffeomorphism from the unit disk onto the complex plane $\mathbb{C}$ with its flat metric. Later $\mathrm{R}$. Schoen $[\mathrm{S}]$ provided a more geometric and simpler argument for a more general situation, a corollary of which is that there is no harmonic diffeomorphism from $\mathbb{H}$ onto a complete surface of nonnegative curvature. One interesting test case to the above question is the converse: can one map the complex plane $\mathbb{C}$ onto the Poincare disk $\mathbb{H}$ by a harmonic diffeomorphism?

Proper harmonic maps between hyperbolic spaces have many nice and interesting properties. See, for example, the works of Li and Tam [LT1, LT2, LT3], Akutagawa [A], and Akutagwa, Nishikawa and Tachikawa [ANT1, ANT2]. Harmonic maps from a surface into a negatively curved surface also have very rich geometries. For example, there is a connection between harmonic maps from a surface into $\mathbb{H}$ and spacelike constant mean curvature surfaces in the Minkowski space $\mathbb{E}^{2,1}$ through a theorem of T. Milnor [M], which says that the Gauss map of a spacelike constant mean curvature surface in $\mathbb{E}^{2,1}$ is a harmonic map from the surface into $\mathbb{H}$. In [CT2], Choi and Treibergs constructed many entire spacelike constant mean curvature surfaces in $\mathbb{E}^{2,1}$. The images of the Gauss maps of these surfaces in $\mathbb{H}$ are convex hulls of closed sets on the ideal boundary of $\mathbb{H}$. In general, the conformal type of the surface is not easy to be determined. However, if the image of the Gauss map is the convex hull of a finite number $\geq 3$ points on the ideal boundary of $\mathbb{H}$, then the surface has finite total curvature and is of the conformal type of $\mathbb{C}$. Thus their construction gives examples of harmonic diffeomorphisms from $\mathbb{C}$ into ideal convex polygons in $\mathbb{H}$. In the case the boundary set on the ideal boundary of $\mathbb{H}$ contains an open interval, they can construct constant mean curvature surfaces of the conformal type of $\mathbb{H}$.

Another quantity which describes the geometry of a harmonic map between surfaces is a quadratic differential associated with a harmonic map, called the Hopf differential. It is a fact that this differential is holomorphic for a harmonic map [CG]. It was proved in [WA] that given a holomorphic quadratic differential $\Phi=\phi d z^{2}$ in $\mathbb{C}$, there is an orientation preserving harmonic diffeomorphism from $\mathbb{C}$ into $\mathbb{H}$ with $\Phi$ as its Hopf differential, provided $\phi$ is not a constant. Note that if $\phi$ is a constant, then one can still construct a harmonic map with $\Phi$ as its Hopf differential, but the harmonic 
map fails to be a diffeomorphism. In any case, the constructed harmonic maps can be realized as the Gauss maps of constant mean curvature cuts in the Minkowski three space $\mathbb{E}^{2,1}$. The result of [WA] is generalized in [TW] to the case that the target is a complete, simply connected surface with nonpositive bounded Gaussian curvature, and with positive first eigenvalue.

In this paper, we will discuss harmonic diffeomorphisms from $\mathbb{C}$ into a complete simply connected surface $N$ such that the Gaussian curvature $K_{N}$ of $N$ is bounded between two negative constants. We will address the case when the associated Hopf differential $\phi$ is a polynomial. This case can be considered as a first step to understand the open problem whether there is a harmonic diffeomorphism from $\mathbb{C}$ onto $\mathbb{H}$. We will use a combination of the approaches mentioned above. First, in section 1 we will prove that if the Hopf differential $\Phi=\phi d z^{2}$ of a harmonic diffeomorphism $u$ from $\mathbb{C}$ into $N$ is such that $\phi$ is a polynomial of degree $m$, then the integral of $K_{N}$ over the image of $u$ is exactly $-m \pi$. By abuse of notation, we say that the differential $\Phi=\phi d z^{2}$ is polynomial if $\phi$ is a polynomial.

We will also prove the converse. Namely, if $u$ is a harmonic diffeomorphism from $\mathbb{C}$ into $N$ such that $\|\partial u\|^{2}|d z|^{2}$ is complete and that the integral of $K_{N}$ over the image of $u$ is $-m \pi$, then $m$ is a positive integer and the Hopf differential of $u$ is a polynomial of degree $m$. Here $\|\partial u\|^{2}$ is the $\partial$-energy density of $u$. Combining these two results, if $N=\mathbb{H}$, then we can conclude that the Hopf differential is a polynomial of degree $m$ if and only if the image of the harmonic map is an ideal polygon with $m+2$ vertices on the ideal boundary of $\mathbb{H}$. In fact, this is true even if $N$ is only a simply connected surface with curvature pinched between two negative constants.

We will also answer a uniqueness question. In $[\mathrm{S}]$, Schoen asked the following question: Is a harmonic diffeomorphism from $\mathbb{C}$ onto an ideal polygon of the Poincaré disk unique up to composition with a conformal automorphism of $\mathbb{C}$ ? In section 2 we will show that, generically, the answer is negative. In fact, for $m \geq 3$, we will construct an $m-3$ real parameter family of nontrivially distinct harmonic diffeomorphisms from $\mathbb{C}$ onto a fixed ideal polygon in $\mathbb{H}$ with $m$ vertices. We will show these are all possible harmonic diffeomorphisms with the fixed ideal polygon as image.

Geometric behavior of harmonic maps between compact hyperbolic surfaces has been studied in [Wo] and [My]. By adapting some of their ideas, as done in [Ha], we will show in section 3 that if the Hopf differential of a harmonic diffeomorphism from $\mathbb{C}$ into $N$ is a polynomial, then near infinity, the image of a horizontal trajectory of the Hopf differential is asymptotically a geodesic in $N$. See $\S 3$ for the precise meaning of this statement. We will prove that if the Hopf differential is a polynomial, then the image of a har- 
monic diffeomorphism from $\mathbb{C}$ into $N$ is the convex hull of the intersection of the closure of its image and the ideal boundary of $N$. This gives another proof and also a generalization of the result in [CT2] for the case that the Hopf differential is a polynomial. It should be mentioned that using the idea of trajectories of the Hopf differential, the first author proved part of the results mentioned above [Ha]. Namely, he proved that the image of a harmonic diffeomorphism from $\mathbb{C}$ into $\mathbb{H}$ with polynomial Hopf differential is a polygon.

The authors would like to thank Shiu-Yuen Cheng, Peter Li and Richard Schoen for some useful discussions. This project is done partially during the visit of the first and the fourth authors at Stanford University, invited by Richard Schoen. They would like to thank his support and interest in the work.

\section{Harmonic diffeomorphisms with polynomial Hopf differentials.}

In this section, we will give a characterization for a harmonic diffeomorphism from $\mathbb{C}$ into $\mathbb{H}$ to have a polynomial Hopf differential. More generally, we will study harmonic diffeomorphisms from $\mathbb{C}$ into a complete surface with nonpositive curvature. First, let us recall the definition and some properties of harmonic maps between surfaces. Let $\Sigma_{1}$ and $\Sigma_{2}$ be two Riemann surfaces with conformal metrics $\rho^{2}(z)|d z|^{2}$ and $\sigma^{2}(u)|d u|^{2}$ respectively. The harmonic map equation for maps from $\Sigma_{1}$ into $\Sigma_{2}$ can be written as

$$
u_{z \bar{z}}+2(\log \sigma)_{u} u_{z} u_{\bar{z}}=0 .
$$

Define $\|\partial u\|^{2}=\frac{\sigma^{2}}{\rho^{2}}\left|u_{z}\right|^{2}$, and $\|\bar{\partial} u\|^{2}=\frac{\sigma^{2}}{\rho^{2}}\left|u_{\bar{z}}\right|^{2}$. The energy density of $u$ is given by $e(u)=\|\partial u\|^{2}+\|\bar{\partial} u\|^{2}$, and the Jacobian of the map $u$ is $J(u)=$ $\|\partial u\|^{2}-\|\bar{\partial} u\|^{2}$. At a point where $\|\partial u\|$ is not zero, we have the following Bochner formula for the harmonic map $u$, see [SY] and [Sa]:

$$
\Delta_{\Sigma_{1}} \log \|\partial u\|=-K_{2}(u) J(u)+K_{1},
$$

where $\Delta_{\Sigma_{1}}$ is the Laplacian of $\Sigma_{1}$, and $K_{i}$ is the Gaussian curvature of $\Sigma_{i}$, for $i=1,2$. The Hopf differential of $u$ is defined as $\phi d z^{2}=\sigma^{2}(u) u_{z} \bar{u}_{z} d z^{2}$. It is well known that if $u$ is harmonic then $\phi d z^{2}$ is a holomorphic quadratic differential defined on $\Sigma_{1}$, see [CG]. Let $u$ be an orientation preserving harmonic diffeomorphism from $\mathbb{C}$ into a complete surface $N$ with Gaussian curvature $K_{N} \leq 0$. Let $|d z|^{2}$ and $\sigma^{2}|d u|^{2}$ be the metrics on $\mathbb{C}$ and $N$ 
respectively. Then the Bochner formula for $u$ can be written as

$$
\begin{aligned}
\Delta_{0} w & =-K_{N} J(u) \\
& =-K_{N}\left(e^{2 w}-|\phi|^{2} e^{-2 w}\right),
\end{aligned}
$$

where $\Delta_{0}$ is the Euclidean Laplacian, $w=\log \|\partial u\|$, and $\phi d z^{2}$ is the Hopf differential of $u$. Since $u$ is an orientation preserving diffeomorphism into $N, J(u)>0$, which implies that $\|\partial u\|>0$ everywhere, and that

$$
e^{2 w}>|\phi| \text {. }
$$

Hence (1.1) holds on the whole complex plane $\mathbb{C}$. We want to prove the following theorem:

Theorem 1.1. Let $u$ be a harmonic diffeomorphism from $\mathbb{C}$ into $\mathbb{H}$ such that $e^{2 w}|d z|^{2}$ is a complete metric on $\mathbb{C}$, where $w=\log \|\partial u\|$. Let $\phi d z^{2}$ be the Hopf differential of $u$. Then the image of $\mathbb{C}$ under $u$ is a generalized geodesic polygon with $m+2$ vertices on the ideal boundary of $\mathbb{H}$ if and only if $\phi$ is a polynomial of degree $m$.

Note that, by [WA], using the fact that $e^{2 w}|d z|^{2}$ is complete on $\mathbb{C}$, the harmonic diffeomorphism $u$ can be realized as the Gauss map of a constant mean curvature cut in the Minkowski three space $\mathbb{E}^{2,1}$.

We will prove some more general results, from which the theorem will follow. Let us first give the following Lemma, which is adapted from [Ha]:

Lemma 1.2. Let $w$ be a solution of

$$
\Delta_{0} w=h\left(e^{2 w}-|\phi|^{2} e^{-2 w}\right)
$$

on $\mathbb{C}$ such that $e^{-2 w}|\phi| \leq 1$, where $\phi$ is a polynomial, not identically zero, and $h$ is a smooth function such that $h \geq a^{2}$ for some constant $a>0$. Then

$$
\lim _{z \rightarrow \infty}\left(w-\frac{1}{2} \log |\phi|\right)=0 \text {. }
$$

In fact, there is a constant $C>0$, such that

$$
0 \leq\left(w-\frac{1}{2} \log |\phi|\right)(z) \leq \exp (-C r(z))
$$

provided $r(z)$ is large enough, where $r(z)$ is the distance of $z$ from the origin with respect to the metric $|\phi||d z|^{2}$. 
Proof. By the assumption that $e^{-2 w}|\phi| \leq 1$, we have $w-\frac{1}{2} \log |\phi| \geq 0$ at those points where $\phi \neq 0$. By [WA], there is a solution $v$ of

$$
\Delta_{0} v=a^{2}\left(e^{2 v}-|\phi|^{2} e^{-2 v}\right)
$$

such that $e^{-2 v}|\phi| \leq 1, e^{2 v}|d z|^{2}$ is complete. By [W], see also [TW], we have $w \leq v$. $\mathrm{By}[\mathrm{Ha}]$

$$
(v-\log |\phi|)(z) \leq \exp (-C r(z))
$$

for some constant $C>0$, provided $r(z)$ is large enough. Since $w \leq v$, the lemma follows.

Theorem 1.3. Let $u$ be an orientation preserving harmonic diffeomorphism from $\mathbb{C}$ into $N$ where $N$ is a complete surface with Gaussian curvature $K_{N} \leq-a^{2}$ for some constant $a>0$. Let $\phi d z^{2}$ be the Hopf differential of $u$. Suppose that $\phi$ is a polynomial with degree $m \geq 1$, then

$$
\int_{u(\mathbb{C})}\left(-K_{N}\right) d V_{N}=m \pi
$$

where $d V_{N}$ is the volume element of $N$. In particular, the area of $u(\mathbb{C})$ is less than or equal to $(m \pi) / a^{2}$.

Proof. Let $B(R)$ be the Euclidean ball with radius $R$ centered at the origin. Since $\phi$ is a polynomial of degree $m \geq 1$, there exists a constant $R_{0}>0$ such that $\phi$ has no zero in $|z|>R_{0}$. Let $w=\log \|\partial u\|$. Since $u$ is an orientation preserving harmonic diffeomorphism from $\mathbb{C}$ into $N$, by the Bochner formula:

$$
\begin{aligned}
\Delta_{0} w & =-K_{N}\left(e^{2 w}-|\phi|^{2} e^{-2 w}\right) \\
& \geq a^{2}\left(e^{2 w}-|\phi|^{2} e^{-2 w}\right),
\end{aligned}
$$

in $\mathbb{C}$, where we have used the assumption that $K_{N} \leq-a^{2}$ and $J(u)>0$, which implies that $e^{-2 w}|\phi| \leq 1$. Since $|\phi(z)| \rightarrow \infty$, as $z \rightarrow \infty$, by Lemma 1.2 ,

$$
\lim _{z \rightarrow \infty}\left(w-\frac{1}{2} \log |\phi|\right)=0 .
$$

Let $z_{1}, \cdots, z_{m}$ be the zeroes of $\phi$ (counted with multiplicity), then

$$
\Delta_{0}\left(\frac{1}{2} \log |\phi|\right)=\sum_{i=1}^{m} \pi \delta_{z_{i}}
$$


in the distributional sense, where $\delta_{z_{i}}$ is the point mass at $z_{i}$. Thus for $R>R_{0}$,

$$
\begin{aligned}
m \pi & =\int_{B(R)} \Delta_{0}\left(\frac{1}{2} \log |\phi|\right) \\
& =\int_{\partial B(R)} \frac{\partial}{\partial R}\left(\frac{1}{2} \log |\phi|\right) .
\end{aligned}
$$

Combining with (1.1), we have, for $R>R_{0}$,

$$
\begin{aligned}
\int_{u(B(R))}\left(-K_{N}\right) d V_{N}-m \pi & =\int_{B(R)}\left(-K_{N}\right) J(u) d x d y-m \pi \\
& =\int_{B(R)} \Delta_{0}\left(w-\frac{1}{2} \log |\phi|\right) \\
& =\int_{\partial B(R)} \frac{\partial}{\partial R}\left(w-\frac{1}{2} \log |\phi|\right) \\
& =2 \pi R \frac{d}{d R} f_{\partial B(R)}\left(w-\frac{1}{2} \log |\phi|\right) .
\end{aligned}
$$

Here $f_{\partial B(R)}$ stands for integral average over $\partial B(R)$. If $\int_{u(C)}\left(-K_{N}\right) d V_{N}<$ $m \pi$, then for all $R>R_{0}$,

$$
\int_{u(B(R))}\left(-K_{N}\right) d V_{N}-m \pi<-\epsilon
$$

for some $\epsilon>0$, which would imply

$$
R \frac{d}{d R} f_{\partial B(R)}\left(w-\frac{1}{2} \log |\phi|\right)<-\epsilon .
$$

Dividing $R$ on both sides of the inequality and integrating implies that

$$
f_{\partial B(R)}\left(w-\frac{1}{2} \log |\phi|\right)<0
$$

for large $R$, which contradicts $w-\frac{1}{2} \log |\phi|>0$. On the other hand, if $\int_{u(C)}\left(-K_{N}\right) d V_{N}>m \pi$, then for sufficiently large $R$,

$$
R \frac{d}{d R} f_{\partial B(R)}\left(w-\frac{1}{2} \log |\phi|\right)>\epsilon
$$

for some $\epsilon>0$. Again dividing $R$ on both sides of the inequality and integrating would imply

$$
f_{\partial B(R)}\left(w-\frac{1}{2} \log |\phi|\right)>\epsilon \log R+C
$$


which contradicts (1.3). This proves $\int_{u(C)}\left(-K_{N}\right) d V_{N}=m \pi$. The second part of the theorem then follows from the fact that $-K_{N} \geq a^{2}$.

Remark. Let $u$ be a harmonic map from $\mathbb{C}$ into a complete surface $N$ with Gaussian curvature pinched between two negative constants, such that $\|\partial u\|^{2}|d z|^{2}$ is complete and $\|\partial u\| \geq\|\bar{\partial} u\|$. If the Hopf differential of $u$ is $c d z^{2}$ for some non-zero constant $c$, then $u(\mathbb{C})$ degenerates into a geodesic in $N$. In fact, in this case one can conclude that $\|\partial u\|$ is a constant, see [TW]. Hence $\|\bar{\partial} u\|$ is a constant and so is the energy density. As in [Ht], the image of $u$ is a geodesic.

Theorem 1.4. Let $u$ be an orientation preserving harmonic diffeomorphism from $\mathbb{C}$ into a complete surface $N$ with Gaussian curvature $K_{N} \leq 0$, such that $e^{2 w}|d z|^{2}$ is a complete metric on $\mathbb{C}$, where $w=\log \|\partial u\|$. Let $\phi d z^{2}$ be the Hopf differential of $u$. Let $\int_{u(\mathbb{C})}\left(-K_{N}\right) d V_{N}=m \pi$ for some $0 \leq m \leq \infty$. If $m<\infty$, then $\phi$ is a polynomial with degree $\leq m$. If we assume, in addition, that $K_{N} \leq-a^{2}$ for some $a>0$, then $m$ is the degree of $\phi$, and thus an integer.

Proof. If $m<\infty$, then $\mathbb{C}$ with conformal metric $e^{2 w}|d z|^{2}$ is a complete surface of finite total curvature with nonpositive Gaussian curvature. The first part of the theorem can be deduced from the results in [F]. Here we will give a much simpler and more direct proof.

First, we argue that $\phi$ has only finitely many zeroes. The argument again follows by integrating the Bochner formula. If $\phi$ has more than $[m]+1$ zeroes, then for all sufficiently large $R$ for which $\partial B(R)$ does not contain any zero of $\phi$,

$$
R \frac{d}{d R} f_{\partial B(R)}\left(w-\frac{1}{2} \log |\phi|\right)<-\epsilon
$$

for some $\epsilon>0$. Since the zeroes of a holomorphic functions are discrete, this inequality holds for all large $R$ except a possibly countable set of $R$ 's. Note that $f_{\partial B(R)}\left(w-\frac{1}{2} \log |\phi|\right)$ is continuous in $R$, so that by dividing $R$ and integrating, we again obtain a contradiction with $w-\frac{1}{2} \log |\phi|>0$. Next we prove an upper bound for $w$ in the form $e^{2 w(z)} \leq a_{1}|\phi|^{2}|z|^{b_{1}}$, for all $z$ with large $|z|$, with some constants $a_{1}, b_{1}>0$. It is elementary to see that if $\phi$ is not a polynomial, then there is a curve in $\mathbb{C}$ diverging to $\infty$ with finite length in the metric $|\phi|^{2}|z|^{b_{1}}|d z|^{2}$ (see, for instance, Lemma 9.6 
in [O]), which would imply that $e^{2 w}|d z|^{2}$ is not complete. The derivation of the claimed upper bound follows from the mean value inequality for the subharmonic function $w-\frac{1}{2} \log |\phi|$. As in the proof of Theorem 1.3, we first obtain an upper bound for the spherical average of $w-\frac{1}{2} \log |\phi|$ :

$$
f_{\partial B(R)}\left(w-\frac{1}{2} \log |\phi|\right) \leq \epsilon \log R+c
$$

for some constants $\epsilon, c>0$. Notice that $w-\frac{1}{2} \log |\phi|>0$ is subharmonic, therefore, for any $z$ with sufficiently large $|z|$,

$$
\begin{aligned}
\left(w-\frac{1}{2} \log |\phi|\right)(z) & \leq f_{B(z,|z| / 2)}\left(w-\frac{1}{2} \log |\phi|\right) \\
& \leq \frac{4}{\pi|z|^{2}} \int_{B(3 / 2|z|) \backslash B(|z| / 2)}\left(w-\frac{1}{2} \log |\phi|\right) \\
& \leq \frac{4}{\pi|z|^{2}} \int_{|z| / 2}^{3 / 2|z|}(\epsilon \log R+c) R d R \\
& \leq b_{1} \log |z|+a_{1}
\end{aligned}
$$

for some constants $a_{1}, b_{1}>0$. Exponentiating both sides gives us the desired upper bound. Thus we conclude that $\phi$ is a polynomial with degree less than or equal to $m$.The second statement of the Theorem follows from Theorem 1.3 .

Combining Theorem 1.3 and Theorem 1.4, we have:

Corollary 1.5. Let $u$ be an orientation preserving harmonic diffeomorphism from $\mathbb{C}$ into a complete simply connected surface $N$ with its Gaussian curvature $K_{N}$ bounded from above by a negative constant $-a^{2}$. Suppose that $e^{2 w}|d z|^{2}$ is a complete metric on $\mathbb{C}$, where $w=\log \|\partial u\|$. Let $\phi d z^{2}$ be the Hopf differential of $u$. Then $\phi$ is a polynomial of degree $m$ if and only if $\int_{u(\mathbb{C})}\left(-K_{N}\right) d V_{N}=m \pi$. In particular, under the additional assumption that $K_{N}$ is also bounded below by a constant $-b^{2}$, then $\phi$ is a polynomial if and only if $u(\mathbb{C})$ has finite area.

We are now ready to give the

Proof of Theorem 1.1. By Corollary 1.5, and the fact that the Gaussian curvature of $\mathbb{H}$ is -1 , we can conclude that $\phi$ is a polynomial of degree $m$ if and only if the area of $u(\mathbb{C})$ is $m \pi$. By [WA], using the fact that $e^{2 w}|d z|^{2}$ 
is a complete metric on $\mathbb{C}, u$ can be realized as a constant mean curvature cut in $\mathbb{E}^{2,1}$. By Theorem 4.8 in [CT2], see also Proposition 2.1 in $\S 2$, there is a closed subset $L$ in the ideal boundary of $\mathbb{H}$ such that $u(\mathbb{C})$ is equal to the convex hull of $L$. From the geometry of $\mathbb{H}$, the area of $u(\mathbb{C})$ is $m \pi$ if and only if $L$ consists of exactly $m+2$ points. The theorem then follows.

Remark. A key ingredient in the proof of Theorem 1.1 is Theorem 4.8 in [CT2], which asserts that $u(\mathbb{C})$ is equal to the convex hull of $L$ for a closed subset $L$ in the ideal boundary of $\mathbb{H}$. This is proved in [CT2] by studying the Gauss map of constant mean curvature cuts in $\mathbb{E}^{n, 1}$. That method does not generalize to the case of a negatively curved target surface. In section 3 , by a more intrinsic geometric approach of harmonic maps, we will prove that Theorem 1.1 is still true if $\mathbb{H}$ is replaced by a simply connected surface with Gaussian curvature pinched between negative constants.

In Theorem 1.3 the integral estimate $\int_{u(\mathbb{C})}\left(-K_{N}\right) d V_{N}=m \pi$ does not involve the upper bound of $K_{N}$ in a quantitative way. It seems reasonable that the assumption on $K_{N}$ can be relaxed to $K_{N} \leq 0$. In some particular cases, this can be done. More precisely, in [TW], it was proved that given a holomorphic quadratic differential $\phi d z^{2}$ in $\mathbb{C}$, one can construct a harmonic diffeomorphism $u$ from $\mathbb{C}$ into a complete simply connected surface $N$ with positive first eigenvalue, and with the Gaussian curvature bounded between $-b^{2}$ and 0, such that the Hopf differential of $u$ is $\phi d z^{2}$ and that $\|\partial u\|^{2}|d z|^{2}$ is complete. In fact, $u$ is the limit of a sequence of harmonic maps $u_{i}$ from $\mathbb{C}$ into $N_{i}$ with Hopf differential $\phi d z^{2}$. Here each $N_{i}$ is a simply connected surface with Gaussian curvature pinched between two negative constants. Moreover, each $N_{i}$ can be realized as $\left(\mathbb{D}, e^{2 \lambda_{i}} d s_{p}^{2}\right)$ where $d s_{p}^{2}$ is a Poincaré metric on $\mathbb{D}$, and $\lambda_{i} \rightarrow \lambda$ where $e^{2 \lambda} d s_{p}^{2}$ is the metric of $N$. Using this information and Theorem 1.3 and Theorem 1.4, we can conclude that:

Proposition 1.6. Let $N$ be a simply connected surface with Gaussian curvature $K_{N}$ satisfying $-b^{2} \leq K_{N} \leq 0$ and with positive first eigenvalue. Given any polynomial $\phi$ of degree $m \geq 1$ on $\mathbb{C}$, there is a harmonic diffeomorphism $u$ from $\mathbb{C}$ into $N$ with Hopf differential $\phi d z^{2}$ such that

$$
\int_{u(\mathbb{C})}\left(-K_{N}\right) d V_{N}=m \pi
$$




\section{Constant mean curvature cuts.}

In [S], Schoen asked the following question: Is a harmonic diffeomorphism from $\mathbb{C}$ onto an ideal polygon of the Ponincaré disk unique up to composition with a conformal automorphism of $\mathbb{C}$ ? By [WA], see also [TW], given a polynomial $\phi$ of degree $m$, there is an orientation preserving harmonic diffeomorphism $u$ from $\mathbb{C}$ into $\mathbb{H}$, with Hopf differential $\phi d z^{2}$, such that $\|\partial u\|^{2}|d z|^{2}$ is complete. The map is unique up to isometry in $\mathbb{H}$ By the results in $\S 1$, we see that the image of the harmonic map is an ideal polygon with $m+2$ vertices. Hence we can establish a correspondence between the set of polynomials of degree $m$ and the set of ideal polygons in $\mathbb{H}$ with $m+2$ vertices. By composition with conformal automorphism of $\mathbb{C}$, we may consider only the set of polynomials such that the coefficient of the leading term is 1 , and the constant term is 0 . Hence that is $2 m-2$ real parameter family of polynomials. An ideal polygon with $m+2$ sides in $\mathbb{H}$ is determined by the $m+2$ vertices. By composition with an isometry of $\mathbb{H}$, we may assume that three of the vertices are $1,-1$, and $i$. The family of such polygons has $m-1$ parameters. Hence generically, for any fixed $m+2$ points on the ideal boundary of $\mathbb{H}$, there should be an $m-1$ parameter family of harmonic diffeomorphisms such that the image of each of them is the ideal polygon with those $m+2$ points as vertices. In this section, we will show that that is the case for every set of $m+2$ points. In fact, we will construct all harmonic diffeomorphisms $u$ with a fixed ideal polygon as image, such that $\|\partial u\|^{2}|d z|^{2}$ is complete. Equivalently, we will find all parabolic constant mean curvature cuts with a fixed ideal polygon as the Gauss image.

First, let us review some results in constant mean curvature cuts in [CT1, CT2, CT3]. Three dimensional Minkowski, $\mathbb{E}^{2,1}$, is $\mathbb{R}^{3}$ endowed with the Lorentz metric

$$
d s^{2}=d x_{1}{ }^{2}+d x_{2}{ }^{2}-d x_{3}{ }^{2} .
$$

A smooth surface $M \subset \mathbb{E}^{2,1}$ is spacelike if the restriction of $d s^{2}$ to $M$ is a Riemannian metric. If the surface is given locally as a graph $x_{3}=f(x)$ where $x=\left(x_{1}, x_{2}\right)$ then the induced metric has the form

$$
\langle\cdot, \cdot\rangle=d s^{2}=g_{i j} d x_{i} d x_{j}, \quad g_{i j}=\delta_{i j}-f_{i} f_{j} .
$$

Thus, being spacelike is equivalent to $|D f|<1$.

A spacelike surface $M \subset \mathbb{E}^{2,1}$ is entire if the projection $M \ni\left(x, x_{3}\right) \mapsto x$ is onto $\mathbb{R}^{2}$, where $x=\left(x_{1}, x_{2}\right)$. If it is also closed with respect to the ambient topology we call it a cut. It is well-known that if $M$ is a constant mean curvature cut then $M$ is the graph of a function $f$ defined over the 
entire $\mathbb{R}^{2}$, see [CT2] for example. Let $\left\{\mathbf{e}_{1}, \mathbf{e}_{2}, \mathbf{e}_{3}\right\}$ be an orthonormal frame of $\mathbb{E}^{2,1}$ adapted to $M$, that is, $\mathbf{e}_{3}$ is the future pointing normal vector field. The set of all future pointing vectors of $\mathbb{E}^{2,1}$ of length -1 is a hyperboloid $\mathcal{H}$ which is isometric to that Poincaré disk in the induced metric. The map $\mathcal{G}: M \rightarrow \mathcal{H}$ taking a point to its unit normal translated to the origin gives the Gauss map

$$
\mathcal{G}(x)=\mathbf{e}_{3}=\left(\frac{D f}{\sqrt{1-|D f|^{2}}}, \frac{1}{\sqrt{1-|D f|^{2}}}\right) .
$$

The isometry of $\mathcal{H}$ to $\mathbb{H}=\left(\mathbb{D}, d s^{2}\right)$ where $\mathbb{D}=\{z \in \mathbb{C}:|z|<1\}$ and $d s^{2}$ is the Klein metric, is given by $\left(x_{1}, x_{2}, x_{3}\right) \mapsto\left(x_{1} / x_{3}, x_{2} / x_{3}\right)$ so the Gauss map becomes $\mathcal{G}(x)=D f(x): M \rightarrow \mathbb{H}$, see [CT2]. The geometric boundary coincides with the usual topological one $\mathbb{H}(\infty)=\partial \mathbb{D}$.

The second fundamental form is gotten by covariantly differentiating the Gauss map $h_{i j}=\left\langle\nabla_{\mathbf{e}_{i}} \mathbf{e}_{j}, \mathbf{e}_{3}\right\rangle$. The mean curvature $H$ is given by

$$
H=\frac{1}{2} \operatorname{div}\left(\frac{D f}{\sqrt{1-|D f|^{2}}}\right)=\frac{h_{11}+h_{22}}{2} .
$$

We summarize some facts about constant mean curvature cuts in the following proposition [CT2, T2].

Proposition 2.1. Let $M \subset \mathbb{E}^{n, 1}$ be an entire spacelike hypersurface whose mean curvature $H$ is constant and which is closed in the ambient topology. Then

(i) $M$ is complete [CY]. (In fact, if a closed spacelike constant mean curvature surface is complete then it is also entire [W].)

(ii) The length of the second fundamental form is bounded, [C, CY]. In fact, we have $\sum_{i, j=1}^{n} h_{i j}^{2} \leq n^{2} H^{2}$. This implies the Bernstein property: $H=0$ cuts of any dimension are affine subspaces.

(iii) If $H>0$, then $M$ is convex: $h_{i j} \geq 0[C Y, T 1]$. If $n=2$, by (ii), the Gaussian curvature satisfies $-2 H^{2} \leq K \leq 0$.

(iv) There is a splitting phenomenon [M, CT2]. The matrix $\left(h_{i j}\right)$ has constant rank. If $\mathrm{e}_{1}\left(x_{0}\right)$ is a null direction of $h_{i j}$ at a point then $M$ metrically splits a line, e. $g$., if $n=2$ then, up to a rotation, $M=\mathbb{R}^{1} \times N$ where $N \subset \mathbb{E}^{1,1}$ is a spacelike constant curvature curve (a hyperbola). 
(v) By convexity and achronicity, blowing down converges to a convex achronal homogeneous function, the projective boundary data

$$
V_{f}(x)=\lim _{\substack{r \rightarrow \infty \\ r>0}} \frac{f(r x)}{r}
$$

which is lightlike in the following sense [T1]: there is a closed set, the set of lightlike directions $L_{f} \subset \mathbb{S}^{1}$ so that $V_{f}(x)=\sup \{\xi \bullet x: \xi \in$ $\left.L_{f}\right\}$ where "•" is the usual $\mathbb{R}^{2}$ inner product. It follows [CT2] that $\mathcal{G}(M)=\operatorname{Conv}\left(L_{f}\right)$, the convex hull in $\mathbb{H}$. In fact by (iii) and (iv), if there are more than two points in $L_{f}$ then $\mathcal{G}: M \rightarrow \mathcal{G}(M)$ is a diffeomorphism.

(vi) The Gauss map is harmonic [M, I, CT2]. This is the Minkowski Space version of the Ruh-Vilms result.

There is a corresponding existence theory. We define for each $\theta \in \mathbb{S}^{n-1}$ the asymptotic boundary values to be

$$
\Lambda_{f}(\theta):=\lim _{r \rightarrow \infty}(f(r \theta)-r)
$$

which decreases either to a limit or to $-\infty$.

Proposition 2.2. [CT2, CT3] Let $L \subset \mathbb{S}^{n-1}$ be any closed set with at least two points and $t(\theta)$ be an upper semicontinuous function defined on $L$. Then there exists a unique entire closed spacelike constant mean curvature $H>0$ surface in Minkowski space, $f \in C^{\omega}\left(\mathbb{R}^{n}\right)$ satisfying (2.1) such that $V_{f}(x)=\sup \{\xi \bullet x: \xi \in L\}$ and $t(\theta)=\Lambda_{f}(\theta)$ for all $\theta \in L$.

Sketch of proof for the present case that $n=2$ and $L$ is finite. For $m \geq 2$ let $L=\left\{\theta_{1}, \theta_{2}, \ldots, \theta_{m}\right\} \subset \mathbb{S}^{1}$ be distinct points taken sequentially around the circle and $t=\left(t_{1}, \ldots, t_{m}\right) \in \mathbb{R}^{m}$. We seek a unique spacelike entire solution $f$ to (2.1) which satisfies the boundary conditions

$$
V_{f}(x)=\max _{k=1, \ldots, m} x \bullet \theta_{k} \quad \Lambda_{f}(\theta)=\left\{\begin{array}{ll}
t_{k}, & \text { if } \theta=\theta_{k}, \\
-\infty, & \text { otherwise. }
\end{array} .\right.
$$

Our method is based on the construction of supersolutions and subsolutions which are constructed from (2.2) alone. These upper and lower solutions, say $\hat{u} \geq \check{u}$, respectively, on $\mathbb{R}^{2}$ will have the property that

$$
\lim _{r \rightarrow \infty}(\hat{u}(r \theta)-\check{u}(r \theta))=0
$$


for all $\theta \in \mathbb{S}^{1}$.

The basic building blocks for barrier construction are surfaces of revolution [HN, CT1, CT2, CT3]. The most basic are the hyperboloid and hyperbolic cylinder

$$
h(x)=\sqrt{1+|x|^{2}}, \quad \tau(x)=\sqrt{\frac{1}{4}+\left|x_{2}\right|^{2}} .
$$

Another is the semitrough which is asymptotic as $|x| \rightarrow \infty$ to the hyperboloid for $x_{1} \leq 0$ and the cylinder for $x_{1} \geq 0$. It has the parametric form

$$
\mathbb{R}^{2} \ni(u, v) \mapsto\left(u-\frac{1}{2} \operatorname{coth} u, \frac{1}{2} \operatorname{coth} u \operatorname{sh} v, \frac{1}{2} \operatorname{coth} u \operatorname{ch} v\right)
$$

Let $x_{3}=\sigma(x)$ denote the graph of this solution and $v(x)=\left|x_{2}\right| . \quad \sigma$ is asymptotic to $|x|$ for $|x| \rightarrow \infty$ with $x_{1} \leq 0$ and $\sigma(x)$ is asymptotic to $\tau$ as $|x| \rightarrow \infty$ with $x_{1} \geq 0$. Hence these solutions satisfy

$$
\begin{array}{ll}
V_{h}(x)=|x|, & \Lambda_{h}(\theta)=0 \\
V_{\tau}(x)=v(x), & \Lambda_{\tau}(\theta)= \begin{cases}0, & \text { if } \theta=(0, \pm 1) \\
-\infty, & \text { otherwise }\end{cases} \\
V_{\sigma}(x)=\left\{\begin{array}{lll}
v(x), & \text { if } x_{1} \geq 0, \\
|x|, & \text { if } x_{1} \leq 0 .
\end{array}\right. & \Lambda_{\sigma}(\theta)= \begin{cases}0, & \text { if } \theta \bullet(1,0) \leq 0, \\
-\infty, & \text { otherwise. }\end{cases}
\end{array}
$$

The key relation is that for $x \in \mathbb{R}^{2}$,

$$
\tau(x)<\sigma_{A}(x)=\sigma\left(x_{1}-A, x_{2}\right)
$$

for any constant $A$ so that

$$
\Lambda_{\tau}(\theta) \leq \Lambda_{\sigma_{A}}(\theta)
$$

for all $\theta$. Under Minkowski Space isometries, this relation continues to hold in the corresponding sector.

Viewing $x \in \mathbb{C}$ let $(R u)(x)=u\left(e^{-i \kappa} x\right)$ denote rotation about the $x_{3}$-axis and $(T u)(x)=u\left(x_{1}-a, x_{2}-b\right)+c$ denote translation. The isometry of boosting by angle $\gamma$ around the $x_{2}$-axis, is

$$
\left(\begin{array}{l}
x_{1} \\
x_{2} \\
x_{3}
\end{array}\right) \longmapsto\left(\begin{array}{c}
x_{1} \operatorname{ch} \gamma+x_{3} \operatorname{sh} \gamma \\
x_{2} \\
x_{1} \operatorname{sh} \gamma+x_{3} \operatorname{ch} \gamma
\end{array}\right)
$$


Let $x_{3}=(B u)(x)$ denote the function whose graph is the boost of the surface $x_{3}=u(x)$. Using definitions, we check that any isometry $\iota$ commutes with blowing down

$$
\left(\iota V_{f}\right)(x)=V_{\iota f}(x) .
$$

On the other hand the asymptotic boundary values transform according to

$$
\begin{aligned}
& \Lambda_{T u}(\theta)=\Lambda_{u}(\theta)-(a, b) \bullet \theta+c \\
& \Lambda_{R u}(\theta)=\Lambda_{u}\left(e^{-i \kappa} \theta\right) \\
& \Lambda_{B u}(\theta)=\operatorname{ch} \gamma \Lambda_{u}\left(e^{i \chi}\right)
\end{aligned}
$$

where $\chi=\chi(\gamma, \theta)$ satisfies

$$
\theta=\frac{\cos \chi \operatorname{ch} \gamma+\operatorname{sh} \gamma+i \sin \chi}{\cos \chi \operatorname{sh} \gamma+\operatorname{ch} \gamma} .
$$

For each $k=1, \ldots, m$, by rotating and boosting it is possible to choose an isometry $B R$ which takes the finite directions $\{ \pm i\}$ of $\Lambda_{\tau}$ to the pair $\left\{t_{k-1}, t_{k}\right\}$ where $\theta_{0}=\theta_{m}$. Hence

$$
V_{B R \tau}(x)=\max \left\{x \bullet \theta_{k-1}, x \bullet \theta_{k}\right\} .
$$

Fixing $a$ and choosing $b$ and $c$ appropriately for the translation $T$ we can arrange that the composite $\iota_{k}=B R T$ takes prescribed values at $\theta=\theta_{k-1}$ and $\theta=\theta_{k}$,

$$
\Lambda_{\iota_{k} \tau}(\theta)= \begin{cases}t_{k-1}, & \text { if } \theta=\theta_{k-1} \\ t_{k}, & \text { if } \theta=\theta_{k}, \\ -\infty, & \text { otherwise. }\end{cases}
$$

Applying the same isometry to $\sigma$ we have

$$
\begin{gathered}
V_{\iota_{k} \sigma}(x)= \begin{cases}\max \left\{x \bullet \theta_{k-1}, x \bullet \theta_{k}\right\}, & \text { if } \arg \theta_{k-1} \leq \arg x \leq \arg \theta_{k} \\
|x|, & \text { otherwise. }\end{cases} \\
\Lambda_{\iota_{k} \sigma}(\theta)= \begin{cases}t_{k-1}, & \text { if } \theta=\theta_{k-1}, \\
t_{k}, & \text { if } \theta=\theta_{k}, \\
-\infty, & \text { if } \arg \theta_{k-1}<\arg \theta<\arg \theta_{k}, \\
\lambda_{k}(\theta), & \text { otherwise, }\end{cases}
\end{gathered}
$$

where $\lambda_{k}(\theta)$ is a continuous function on the closed $\mathbb{S}^{1}$ segment from $\theta_{k}$ to $\theta_{k-1}$. 
The isometry $\iota_{k}$ was determined by the prescription of $\Lambda_{\iota_{k}}$ for $\arg \theta_{k-1} \leq$ $\arg \theta \leq \arg \theta_{k}$ and is independent of $a$. Since there are only finitely many $k$, by choosing $a$ sufficiently large we may also arrange $\iota_{k} \sigma(x)>\iota_{\ell} \tau(x)$ for all $x, k$ and $\ell$. Let

$$
\check{u}(x)=\max _{k=1, \ldots, m} \iota_{k} \tau(x), \quad \hat{u}(x)=\min _{k=1, \ldots, m} \iota_{k} \sigma(x) .
$$

$\check{u}$ and $\hat{u}$ are subsolution and supersolution to (2.1) satisfying the boundary condition (2.2). By approximation [T1] where a local version of Proposition 2.1 (2) provides the compactness estimates, there is an entire constant mean curvature surface $\hat{u}>f>\check{u}$ with $V_{f}=V_{\check{u}}$ and $\Lambda_{f}=\Lambda_{\check{u}}$.

To prove uniqueness, let $u$ be another solution to (2.1) satisfying the boundary condition (2.2). Since any solution is convex by Proposition 2.1 (3), then $x \bullet \theta_{i}+t_{i}$ is a lower barrier for each $i$. Hence

$$
u(x)>q(x):=\sup _{k=1, \ldots, m} x \bullet \theta_{k}+t_{k}
$$

so by achronicity, $u\left(r \theta_{k}\right)-q\left(r \theta_{k}\right) \rightarrow 0$ as $r \rightarrow \infty$. Let $\iota_{k} \tau$ be one of the subsolutions constructed above. By (2.4) $u>\iota_{k} \tau-\varepsilon$ if $\varepsilon$ sufficiently large. In decreasing $\varepsilon, \iota_{k} \tau-\varepsilon$ remains a global subsolution for any solution $u$ and any $\varepsilon>0$. If not, let $\varepsilon_{0}>0$ be the infimum of such $\varepsilon$. Either $u$ touches $\iota_{k} \tau-\varepsilon_{0}$ at an interior point which cannot happen by the strong maximum principle, or by (2.4), it can touch asymptotically at most one possible point at infinity $\lim _{r \rightarrow \infty} u(r \bar{\theta})-\iota_{k} \tau(r \bar{\theta})=-\varepsilon_{0}$ where $\bar{\theta}=\frac{1}{2}\left(\theta_{k-1}+\theta_{k}\right)$. This cannot happen either as can be seen by "blowing up" at $\bar{\theta} \in \mathbb{H}(\infty)$. In fact, if we let $g=\iota_{k}^{-1} u$, then $g>\tau-\varepsilon_{0}^{\prime}$ for some $\varepsilon_{0}^{\prime}>0$, and $\lim _{x_{1} \rightarrow \infty} g\left(x_{1}, 0\right)=\frac{1}{2}-\varepsilon_{0}^{\prime}$. Then the constant mean curvature cut $w\left(x_{1}, x_{2}\right)=\lim _{t \rightarrow \infty} g\left(x_{1}+t, x_{2}\right)$ is the monotonic limit of constant mean curvature cuts which depends only on $x_{2}$, and has boundary data $V_{w}=V_{\tau}$ and $\Lambda_{w}=\lambda_{\tau}$ but $w \neq \tau$. This is impossible by looking at the ordinary differential equation satisfied by both $w$ and $\tau$. Hence we have $u \geq \iota_{k} \tau$ for all $k$. Hence $u \geq \check{u}$.

To prove $\hat{u} \geq u$, let us denote by $\sigma^{\prime}=\sigma\left[\theta_{k-1}, \theta_{k}, t_{k-1}, t_{k}\right]$ the boosted $\sigma$ solution that has its Gauss map image $G^{\prime}$, the convex hull of $\mathbb{S}^{1}-\left(\theta_{k-1}, \theta_{k}\right)$, and $\Lambda_{\sigma^{\prime}}\left(\theta_{i}\right)=t_{i}$ for $i=k-1, k$ and which is minimal among those for which $\sigma^{\prime} \geq q$. If we take a subinterval $\left[\xi_{1}, \xi_{2}\right] \subset\left(\theta_{k-1}, \theta_{k}\right)$ then the solution $\sigma^{\prime \prime}=\sigma\left[\xi_{1}, \xi_{2}, t_{k-1}, t_{k}\right]$ has $G \subset \overline{G^{\prime}} \subset G^{\prime \prime}$ where $G$ is the Gauss image of $u$. Hence $\sigma^{\prime \prime}>f$ in the sense that if $z \in G^{\prime \prime}-G, \theta=z /|z|$, then

$$
\lim _{r \rightarrow \infty} \frac{\sigma(r \theta)}{r} \geq \theta \bullet z>\lim _{r \rightarrow \infty} \frac{u(r \theta)}{r} .
$$


Together with the fact that $u$ is achronal,

$$
\lim _{r \rightarrow \infty} \sigma^{\prime \prime}(r \theta)-u(r \theta) \geq 0 .
$$

Hence also $\sigma^{\prime \prime}>u$ for all $x \in \mathbb{R}^{2}$. Now, as for subsolutions, $\iota_{k} \sigma$ is the infimum of such $\sigma^{\prime \prime}$. Hence $\iota_{k} \sigma \geq u$, and $\hat{u} \geq u$. Since $\hat{u}-\check{u}$ tends to 0 as $x \rightarrow \infty$, and $\hat{u} \geq u, f \geq \check{u}, u=f$ by the maximum principle.

The constant mean curvature cuts are Hadamard surfaces whose behavior lies between $\mathbb{C}$ and $\mathbb{H}$. If $L_{f}$ contains an interval where $\Lambda_{f}$ is uniformly bounded, then $M$ is conformally the disk [Mi]. (For Lipschitz continuous $\Lambda_{f}$ see [CT3].) Hence there is a uniformizing map $\Upsilon: \mathbb{H} \rightarrow M$ so that $\mathcal{G} \circ \Upsilon: \mathbb{H} \rightarrow \mathcal{G}(M)$ is harmonic.

On the other hand, for finite $L$, by (iv), $\mathcal{G}(M)=\mathbb{P}$ is a polygon. Since the cut is complete and has finite total curvature (their Gauss image is diffeomorphic to a polygon with finite area), then it must be parabolic according to the theorem of Blanc and Fiala $[\mathrm{BF}]$ and Huber [Hu]. Hence there is a uniformizing map $\Upsilon: \mathbb{C} \rightarrow M$ so that $u=\mathcal{G} \circ \Upsilon: \mathbb{C} \rightarrow \mathbb{P}$ is harmonic. We say that two harmonic maps $u_{1}, u_{2}: \mathbb{C} \rightarrow \mathbb{P}$ are nontrivially distinct if there is no conformal diffeomorphism $w: \mathbb{C} \rightarrow \mathbb{C}$ so that $u_{2}=u_{1} \circ w$.

Theorem 2.3. Let $\mathbb{P} \subset \mathbb{H}$ be an ideal polygon in the hyperbolic space with $m \geq 3$ vertices $\theta_{k} \in \mathbb{H}(\infty)$. For each $t \in \mathbb{R}^{m}$ let $M(t) \subset \mathbb{E}^{2,1}$ be the entire spacelike surface of constant mean curvature $H=1$ whose Gauss image $\mathcal{G}(M(t))=\mathbb{P}$ and $\Lambda_{M(t)}\left(\theta_{k}\right)=t_{k}$, which uniquely exists by Proposition 2.2. Let $N \subset \mathbb{R}^{m}$ be the three dimensional subspace of restrictions of affine functions,

$$
N=\left\{s \in \mathbb{R}^{m}: s_{k}=(a, b) \bullet \theta_{k}+c, k=1, \ldots, m, \quad \text { some }(a, b, c) \in \mathbb{R}^{3}\right\}
$$

Then for each class in $[t] \in \mathbb{R}^{m} / N$ the harmonic maps

$$
u(\cdot ; t)=\mathcal{G}(\Upsilon(\cdot ; t) ; t): \mathbb{C} \rightarrow \mathbb{P}
$$

are nontrivially distinct.

Proof. Observe that we do not get more maps. For if $t-\tilde{t} \in N$ then there is a translation $T$ so that $\Lambda_{T \circ M}=\Lambda_{\tilde{M}}$. However, by uniqueness in Proposition 2.2, $T \circ M=\tilde{M}$. Hence $\tilde{\Upsilon}$ and $T \circ \Upsilon$ are uniformizing the same surface. Therefore $u=\tilde{\Upsilon}^{-1} \circ T \circ \Upsilon: \mathbb{C} \rightarrow \mathbb{C}$ is a conformal diffeomorphism giving the equivalence $\mathcal{G} \circ \Upsilon=\mathcal{G} \circ \tilde{\Upsilon} \circ u$. 
On the other hand suppose that the two surfaces $M(t)$ and $M(\tilde{t})$ with uniformizations $\Upsilon(\cdot, t): \mathbb{C} \rightarrow M(t)$ have equivalent harmonic maps. Then we will show that these surfaces must differ by rigid motion and so $t-\tilde{t} \in N$ by (2.4). Thus we may assume that, for all $\zeta \in \mathbb{C}$,

$$
\mathcal{G}(\Upsilon(\zeta ; t) ; t)=\mathcal{G}(\Upsilon(\zeta ; \tilde{t}) ; \tilde{t}) .
$$

Consider the conformal diffeomorphism $\Phi: M(t) \rightarrow M(\tilde{t})$ defined by $\Phi=$ $\tilde{\Upsilon} \circ \Upsilon^{-1}$. By (2.6) we also have $\Phi=\mathcal{G}^{-1} \circ \mathcal{G}$ so that $M$ and $\tilde{M}$ are parallel at corresponding points. Let $\left\{\mathbf{e}_{i}\right\}$ be an adapted frame near $x \in M$ and its translation the frame for $\tilde{M}$ near $\Phi(x)$. Let $\left\{\theta^{i}\right\}$ be the dual frame for $M$ near $x$ and $\left\{\tilde{\theta}^{i}\right\}$ the dual frame near $\Phi(x)$. Pulling back, we obtain functions

$$
\phi_{j}^{i} \theta^{j}=\Phi^{*} \tilde{\theta}^{i} .
$$

Conformality means for some function $\rho>0$,

$$
\rho^{2}\left(\left(\theta^{1}\right)^{2}+\left(\theta^{2}\right)^{2}\right)=\Phi^{*}\left(\left(\tilde{\theta}^{1}\right)^{2}+\left(\tilde{\theta}^{2}\right)^{2}\right)=\phi_{j}^{i} \phi_{k}^{i} \theta^{j} \otimes \theta^{k} .
$$

Hence $\rho^{-1} \phi^{i}{ }_{j}$ is an orthogonal matrix. Thus, if it is also orientation preserving it satisfies

$$
\phi_{1}^{1}=\phi_{2}^{2}, \quad \phi_{2}^{1}=-\phi_{1}^{2} .
$$

But we have the description of $\Phi$ by Gauss maps $\mathcal{G}=\mathbf{e}_{3}$ to the hyperboloid. Another translate of the frame is adapted to the hyperboloid near $\mathbf{e}_{3}$. Denoting the coframe by $\left\{\omega^{A}\right\}$ and connection forms by $\left\{\theta_{i}{ }^{j}\right\}$ we find,

$$
d \mathbf{e}_{3}=\theta_{3}{ }^{i} \mathbf{e}_{i}=\omega^{i} \mathbf{e}_{i}
$$

so that

$$
h_{i j} \theta^{j}=\theta_{3}^{i}=\mathcal{G}^{*} \omega^{j}
$$

Hence, because $\Phi=\tilde{\mathcal{G}}^{-1} \circ \mathcal{G}$ we have

$$
\phi_{k}^{i} \theta^{k}=\Phi^{*} \tilde{\theta}^{i}=\tilde{h}^{i j} h_{j k} \theta^{k}
$$

where $h_{i j}(x)$ is the second fundamental form of $M(t)$ and $\tilde{h}^{i j}(\Phi(x))$ is the inverse of the second fundamental form of $M(\tilde{t})$ which is invertible by Proposition 1.4. Thus $\Phi$ is orientation preserving.

We claim that conformality implies $h_{i j}(x)=\tilde{h}_{i j}(\Phi(x))$. The easiest way to see this is to assume that at a point, the second fundamental form is diagonal $h_{i j}(x)=\operatorname{diag}\left(\kappa_{1}, \kappa_{2}\right)$. Thus (2.7) implies

$$
\tilde{h}^{11} \kappa_{1}=\tilde{h}^{22} \kappa_{2}, \quad \tilde{h}^{12} \kappa_{2}=-\tilde{h}^{21} \kappa_{1} .
$$


First we have $\tilde{h}_{12}=0$. To see this, since $\tilde{h}^{i j}$ is symmetric, the second equation implies $\tilde{h}^{12}\left(\kappa_{1}+\kappa_{2}\right)=2 H \tilde{h}^{12}=0$. Then constant mean curvature and the first equation implies

$$
\frac{\kappa_{1}}{\tilde{h}_{11}}=\frac{2 H-\kappa_{1}}{2 H-\tilde{h}_{11}} .
$$

Hence $\kappa_{1}=\tilde{h}_{11}$ and the claim follows.

(2.8) implies $\phi_{j}^{i}=\delta_{j}^{i}$. Hence $\Phi$ is a diffeomorphism for which the first and second fundamental forms agree at the corresponding points. By the Minkowski Space version of the fundamental theorem of surface theory, the surfaces are translates of one another.

If the ideal polygon has two vertices, by uniqueness in Proposition 2.2 it must be a rigid motion of the cylinder $\iota \tau$. The map $\iota(x, \operatorname{sh} y, \operatorname{ch} y) \mapsto(x, y)$ gives an isometry to $\mathbb{C}$.

We want to show that any harmonic diffeomorphism $u$ from $\mathbb{C}$ into $\mathbb{H}$ with the usual assumption and with polygonal image such that $\|\partial u\|^{2}|d z|^{2}$ is complete, then it can be realized as a constant mean curvature cut in the family constructed in Theorem 2.3. That is to say, for any $m \geq 3$, Theorem 2.3 give all the solutions for harmonic diffeomorphisms $u$ from $\mathbb{C}$ into the hyperbolic space so that $\|\partial u\|^{2}|d z|^{2}$ is complete, and that the Hopf differential of $u$ is $\phi d z^{2}$ with $\phi$ to be a polynomial of degree $m-2$. We always assume the mean curvature to be $H=1$ in the following. According to Proposition 2.2, it is sufficient to show the following property about cuts with polygonal $\mathcal{G}(f)$.

Proposition 2.4. Let $f \subset \mathbb{E}^{2,1}$ be an entire spacelike $H=1$ cut such that

$$
V_{f}(x)=\max _{k=1, \ldots, m} x \bullet \theta_{k}
$$

for some $\theta_{k} \in \mathbb{S}^{1}$. Then $\Lambda_{f}(\theta)=-\infty$ for $\theta \neq \theta_{k}$, and $\Lambda_{f}\left(\theta_{k}\right)$ is finite for $k=1, \ldots, m$.

Proof. Note that by Proposition 2.1(5), if the image is a polygon, then (2.9) holds with $\Theta:=\left\{\theta_{k}\right\}_{k=1, \ldots, m} \subset \mathbb{S}^{1}$ the vertices of the polygon. Let $\theta \in \mathbb{S}^{1}$, then we have

$$
\begin{array}{ll}
V_{f}(\theta)=1 & \text { if } \theta \in \Theta, \\
V_{f}(\theta)<1 & \text { if } \theta \notin \Theta .
\end{array}
$$


By the definition of $\Lambda_{f}$, we see that $\Lambda_{f}\left(\theta_{k}\right)=t_{k}$ for $1 \leq k \leq m$, where $t_{k}$ might be $-\infty$, and $\Lambda_{f}(\theta)=-\infty$ if $\theta \notin \Theta$. Let $s_{k}$ be arbitrary real numbers such that $s_{k}>t_{k}$. By Proposition 2.2, there is $u$ such that the graph of $u$ is a constant mean curvature cut with the boundary conditions

$$
V_{u}(x)=\max _{k=1, \ldots, m} x \bullet \theta_{k}
$$

and

$$
\Lambda_{u}(\theta)= \begin{cases}s_{k}, & \text { if } \theta=\theta_{k}, 1 \leq k \leq m \\ -\infty & \text { otherwise. }\end{cases}
$$

We want to prove, $\Lambda_{f}\left(\theta_{m}\right)$ is finite, for example. Suppose not, then $\Lambda_{f}\left(\theta_{m}\right)=$ $-\infty$. Let

$$
\delta=\max _{1 \leq k \leq m-1} \theta_{m} \bullet \theta_{k} .
$$

Then $\delta<1$. For any $\rho>0$, let $(a, b)=\rho \theta_{m} \in \mathbb{C}$, and let $v\left(x_{1}, x_{2}\right)=$ $u\left(x_{1}-a, x_{2}-b\right)+\delta \rho$. By (2.3) we have

$$
\Lambda_{v}(\theta)=\Lambda_{u}(\theta)-\rho \theta_{m} \cdot \theta+\delta \rho .
$$

Hence if $\theta \neq \theta_{k}$, for all $k$, then $\Lambda_{v}(\theta)=-\infty$, and for $1 \leq k \leq m-1$,

$$
\Lambda_{v}\left(\theta_{k}\right)=\Lambda_{u}\left(\theta_{k}\right)-\rho \theta_{m} \bullet \theta_{k}+\delta \rho \geq s_{k}>t_{k}
$$

by the definition of $s_{k}$ and $\delta$. Also $\Lambda_{v}\left(\theta_{m}\right)>-\infty$. Hence $\Lambda_{v} \geq \Lambda_{f}$. By the proof of Proposition 2.2, we see that the supersolution $\hat{v}$ for constructing $v$ satisfies $\hat{v} \geq f$. Since $\hat{v}-v$ tends to 0 at infinity, $v \geq f$ by the maximum principle. In particular, we have

$$
f\left(\rho \theta_{m}\right)=f(a, b) \leq v(a, b)=u(0,0)+\delta \rho .
$$

Dividing through by $\rho$, and letting $\rho \rightarrow \infty$, we have $1 \leq \delta$, where we have used (2.10). This is a contradiction.

Combining Theorem 2.3 and Proposition 2.4, we have

Corollary 2.5. All orientation preserving harmonic diffeomorphism $u$ from $\mathbb{C}$ into an ideal polygon $\mathbb{P}$ in $\mathbb{H}$ with $\|\partial u\|^{2}|d z|^{2}$ complete on $\mathbb{C}$ can be realized as a constant mean curvature cut in the family constructed in Theorem 2.3 
Proof. First note that any such a harmonic diffeomorphism can be realized as a constant mean curvature cut in $\mathbb{E}^{2,1}$, then use Proposition 2.4 and uniqueness in Proposition 2.2.

As mentioned before, for a constant mean curvature cut in $\mathbb{E}^{2,1}$ which is the graph of the function $f$, suppose that $L_{f}$ contains an interval and that $\Lambda_{f}$ is uniformly bounded from below in that interval, then the cut is conformal to the disk by [Mi]. It is still therefore interesting to see whether $\Lambda_{f}$ is always uniformly bounded from below. If this is true, then one can conclude that there is no harmonic diffeomorphism from $\mathbb{C}$ onto $\mathbb{H}$. However, even the question whether $\Lambda_{f}$ is bounded from below pointwise for every point in $L_{f}$ is still open. Using the method of proof of Proposition 2.4, at least we can prove that:

Proposition 2.6. The subset $\left\{\theta \in L_{f} \mid \Lambda_{f}(\theta)>-\infty\right\}$ is dense in $L_{f}$.

Proof. Suppose $\Lambda_{f}(\theta)=-\infty$ for all $\theta \in\left(\theta_{0}-\epsilon, \theta_{0}+\epsilon\right)$. For any fixed $k \in \mathbb{R}$, the function $t_{k}(\theta)=\max \left\{\Lambda_{f}(\theta), k\right\}$ is an upper semi-continuous function defined on $L_{f}$. Therefore, Proposition 2.2 implies that there exists a function $u$ which graph is a constant mean curvature cut such that

$$
V_{u}(x)=\sup \left\{\xi \bullet x: \xi \in L_{f}\right\}
$$

and

$$
\Lambda_{u}(\theta)= \begin{cases}t_{k}(\theta), & \text { if } \theta \in L_{f}, \\ -\infty, & \text { if } \theta \notin L_{f} .\end{cases}
$$

Now let

$$
\delta=\sup \left\{\xi \bullet \theta_{0}: \xi \in L_{f} \backslash\left(\theta_{0}-\epsilon, \theta_{0}+\epsilon\right)\right\}<1,
$$

and for any $\rho>0$, let $(a, b)=\rho \theta_{0} \in \mathbb{C}$. Consider the function $v\left(x_{1}, x_{2}\right)=$ $u\left(x_{1}-a, x_{2}-b\right)+\delta \rho$ which graph is also a constant mean curvature cut. The rest of the proof is similar to Proposition 2.4.

\section{Geometry of harmonic maps.}

In this section, we will give detailed description about the behavior of the harmonic diffeomorphisms considered in $\S 1$ in terms of the trajectories of holomorphic quadratic differentials. In fact, the original approach that we took to study the mapping behavior of harmonic maps between surfaces was 
to exploit the geometry of a holomorphic quadratic differential associated with the map, see [Ha] for a description. That approach suggested Theorem 1.3 and provided a proof. Although we now have a much simpler proof as presented here in this paper, it is still instructive to see that proof to get a better picture of the geometry of harmonic maps. Moreover, this will give another proof of the result by Choi-Treibergs [CT1] that the image of a harmonic diffeomorphism $u$ from $\mathbb{C}$ into $\mathbb{H}$ is the convex hull of the intersection of the closure of the image with the ideal boundary, for the special case that $\|\partial u\|^{2}|d z|^{2}$ is complete and the Hopf differential of the harmonic map is a polynomial. In fact we will prove more general results by allowing the target to be a complete simply connected surface $N$ with Gaussian curvature bounded between two negative constants.

Let us give a brief summary of the background material on the trajectories of a holomorphic quadratic differential on $\mathbb{C}$. See [St] for more detailed information. Let $\Phi=\phi d z^{2}$ be a holomorphic quadratic differential on $\mathbb{C}$. Then $\Phi$ induces a "measured foliation structure" and a "singular flat metric structure" on $\mathbb{C}$. Consider $|\phi(z) \| d z|^{2}$ as a metric on $\mathbb{C}$. Since $\Phi$ is holomorphic, near a point $z_{0}$ where $\phi\left(z_{0}\right) \neq 0$, we may define $w=w(z)$ by $w(z)=\int_{z_{0}}^{z} \sqrt{\phi(z)} d z$. Here we choose a branch of the square root function. Then $|\phi(z) \| d z|^{2}=|d w|^{2}$, which means that $|\phi(z)||d z|^{2}$ is flat. The preimages of the horizontal lines in the $w$-plane are called the horizontal foliations of $\Phi$, denoted by $\Phi_{h}$; the preimages of the vertical lines in the $w$-plane are called the vertical foliations of $\Phi$, denoted by $\Phi_{v}$. It is easy to see that $\Phi_{h}$ and $\Phi_{v}$ are independent of the choice of the branch of the square root function, so they are canonically associated with $\Phi$. At the zeroes of $\Phi$, these foliations have multiple prong saddle singularities, which metrically correspond to cone-type singularities (with concentrated negative curvature). A horizontal leaf which is not properly contained in another one is called a horizontal trajectory. If a horizontal trajectory is parametrized by $t \in R$, then the half leaf corresponding to $t \geq 0$, or $t \leq 0$ is called a horizontal ray. A horizontal ray is said to be diverging if it leaves any compact set after finite time. Two diverging horizontal rays are said to be in the same horizontal asymptotic direction if there is a conformal coordinate region of the form $\{(x, y): x \geq a, 0 \leq y \leq h\}$, with the two rays corresponding to the top and bottom edges of the region.

Now, let us assume that $\phi$ is polynomial of degree $m \geq 1$. Then the differential $\phi d z^{2}$ has a pole of order $m+4$ near infinity. Hence by well known results, see [St], in a neighborhood $U$ of the infinity in $\mathbb{C}$, there are precisely $m+2$ equivalent horizontal asymptotic directions. We can choose a 
representative in each direction, and label them as $H_{1}, \cdots, H_{m+2}$. Moreover, the two rays of any trajectory which stays in $U$ will tend to infinity in two consecutive directions. In fact, for any two such consecutive directions, there is a family of trajectories which tend to infinity and such that the two rays of each one of the trajectories will tend to infinity in those two consecutive directions. We are ready to study the geometry of a harmonic diffeomorphism from $\mathbb{C}$ into $N$. First we need the following lemma.

Lemma 3.1. Let $N$ be a complete simply connected surface with Gaussian curvature bounded above by $-a^{2}$ for some $a>0$. Let $\gamma$ be a $C^{2}$ curve in $N$ with geodesic curvature bounded by $\epsilon>0$, where $\epsilon<a$. Let $\gamma^{*}$ be the complete geodesic passing through the end points of $\gamma$. Then there is a constant $C>0$ which is independent of $\gamma$ such that $d\left(x, \gamma^{*}\right) \leq C \epsilon$ for all $x$ on $\gamma$.

Proof. Let $\gamma:[0, l] \rightarrow M$ be a $C^{2}$ curve parametrized by arclength. Let $\gamma^{*}$ be the complete unit speed geodesic passing through $\gamma(0)$ and $\gamma(l)$. Let $(u, v)$ be the Fermi coordinates with respect to $\gamma^{*}$ such that $v=0$ is the geodesic $\gamma^{*}$ and $v$ is the distance from the point $(u, v)$ to $\gamma^{*}$. In these coordinates, the metric of $N$ is given by $d s^{2}=f^{2}(u, v) d u^{2}+d v^{2}$. Direct computation gives:

$$
\begin{aligned}
\nabla_{\frac{\partial}{\partial u}} \frac{\partial}{\partial u} & =(\log f)_{u} \frac{\partial}{\partial u}-f f_{v} \frac{\partial}{\partial v} \\
\nabla_{\frac{\partial}{\partial u}} \frac{\partial}{\partial v} & =(\log f)_{v} \frac{\partial}{\partial u} \\
\nabla_{\frac{\partial}{\partial v}} \frac{\partial}{\partial v} & =0 .
\end{aligned}
$$

Let $\kappa$ be the geodesic curvature of $\gamma(t)=(u(t), v(t))$. From these formulas, one can compute to obtain

$$
\kappa^{2}=f^{2}\left(\ddot{u}+\dot{u}^{2}(\log f)_{u}+2 \dot{u} \dot{v}(\log f)_{v}\right)^{2}+\left(\ddot{v}-f f_{v} \dot{u}^{2}\right)^{2}
$$

where " $"$ is the differentiation with respect to $t$. We also have

$$
\begin{aligned}
f_{v v}+K f & =0 \\
f(u, 0) & =1 \\
f_{v}(u, 0) & =0
\end{aligned}
$$

Since $K \leq-a^{2}, f_{v}>0$ for $v>0$. Suppose the maximum $v_{\max }$ of $v$ is attained at $t=0$ or $t=l$, then we have $v_{\max }=0$. Otherwise at some 
interior point $0<t_{0}<l$ where $v$ attains its maximum, $\dot{v}=0$ and $\ddot{v} \leq 0$. Let $\left(u\left(t_{0}\right), v\left(t_{0}\right)\right)=\left(u_{0}, v_{0}\right)$. Here $v_{0}=v_{\max } \geq 0$. Since $\kappa^{2} \leq \epsilon^{2}$, by $(3.1)$

$$
f f_{v} \dot{u}^{2} \leq \epsilon
$$

at $\left(u_{0}, v_{0}\right)$. Since $|\dot{\gamma}|=1, f^{2} \dot{u}^{2}+\dot{v}^{2}=1$, hence at the point $\left(u_{0}, v_{0}\right)$, we have

$$
\frac{f_{v}}{f} \leq \epsilon .
$$

Letting $g=(\log f)_{v}=f_{v} / f$, by (3.2) we have

$$
\begin{aligned}
g_{v} & =-K-g^{2} \\
& \geq a^{2}-g^{2}
\end{aligned}
$$

$g>0$ for $v>0$ and $g(u, 0)=0$. Note that since $g^{2}\left(u_{0}, v_{0}\right) \leq \epsilon^{2}<a^{2}$, $g^{2}\left(u_{0}, v\right)<a^{2}$ for all $0 \leq v \leq v_{0}$. This is because at those points $\left(u_{0}, v\right)$ where $g^{2}<a^{2}, g_{v}>0$. By (3.4), for $0 \leq v \leq v_{0}$,

$$
g\left(u_{0}, v\right) \geq a \tanh (a v)
$$

where we have used the fact that $g\left(u_{0}, 0\right)=0$. By (3.3), we have

$$
a \tanh \left(a v_{0}\right) \leq \epsilon .
$$

Since $\epsilon<a, a v_{0}$ is less than some absolute constant, hence $v_{\max }=v_{0} \leq C \epsilon$ for some constant. Similarly, one can prove that $v_{\min } \geq-C \epsilon$ for some constant, and the lemma follows.

In the following, we always assume that $u$ is an orientation preserving harmonic diffeomorphism from $\mathbb{C}$ into a complete simply connected surface $N$ with Gaussian curvature satisfying $-b^{2} \leq K_{N} \leq-a^{2}$ for some positive constants $a, b$. We also assume that $\|\partial u\|^{2}|d z|^{2}$ is complete on $\mathbb{C}$. Let $\Phi=\phi d z^{2}$ be the Hopf differential of $u$. Then $\Phi$ is holomorphic. We always assume that $\phi$ is a polynomial of degree $m$. As mentioned above, there are $m+2$ asymptotic directions for the horizontal rays of $\Phi$ near infinity. We can choose a representative in each direction and label them as $H_{1}, \ldots, H_{m+2}$.

The relevance of the geometry of $\Phi$ to the harmonic map $u$ is revealed by the following computation: If we use $w=x+i y$ as coordinates on $\mathbb{C}$, where $w=\int^{z} \sqrt{\phi} d z$, so that $|d w|^{2}=|\phi(z)||d z|^{2}$ is our metric on $\mathbb{C}$, then

$$
u^{*}\left(\sigma^{2}(u)|d u|^{2}\right)=(e+2) d x^{2}+(e-2) d y^{2} .
$$


where $\sigma^{2}(u)|d u|^{2}$ is the metric tensor of $N$, and $e$ is the energy density of $u$ with respect to the conformal metric $|\phi \| d z|^{2}$. Thus, $\Phi_{h}$ corresponds to the maximal stretch direction of $d u$ and $\Phi_{v}$ corresponds to the minimal stretch direction of $d u$ at every point on $\mathbb{C}$. In our notation, we have

Lemma 3.2. For any $1 \leq i \leq m+2$, let $H_{i}=\{z(t): t \geq 0\}$. Then $\lim _{t \rightarrow \infty} u(z(t))=\theta_{i}$ for some $\theta_{i}$ in the ideal boundary of $N$.

Proof. For simplicity, we consider the case $i=1$, and assume $H_{1}$ is the curve $z(t), t \geq 0$. Let $w=\log \|\partial u\|$, where $\|\partial u\|^{2}$ is the the $\partial$-energy density of $u$ with respect to the Euclidean metric on $\mathbb{C}$, and let $w_{1}=w-\frac{1}{2} \log |\phi|$. Then $e=2 \cosh \left(2 w_{1}\right)$. Let $\gamma(t)=u(z(t))$. The geodesic curvature of the image $\gamma$ of $\gamma(t)$ can be computed as in [Wo] and [My]:

$$
\kappa(\gamma)=-(e-2)^{1 / 2} \frac{\partial}{\partial y} \log (e+2)^{1 / 2}=-\frac{1}{2}(e-2)^{-1 / 2}(e+2)^{-1} \frac{\partial e}{\partial y}
$$

By Lemma 1.2, if the distance of $z$ to the zeroes of $\phi$ is $R$, then $e(z)-2$ is bounded above by $\exp \left(-C_{1} R\right)$ for some positive constant $C_{1}$, provided $R$ is large enough. Note that we always have $e-2 \geq 0$. By the Bochner formula

$$
\Delta_{|\phi|} w_{1}=2 \sinh \left(2 w_{1}\right)
$$

where $\Delta_{|\phi|}$ is the Laplacian for the metric $|\phi||d z|^{2}$, which is complete near infinity of $\mathbb{C}$. By the standard gradient estimate, we see that $\left|\nabla w_{1}(z)\right| \leq$ $\exp \left(-C_{2} R\right)$ for some positive constant $C_{2}$, where $\nabla$ is the gradient with respect to the $\phi$-metric. Hence as $t \rightarrow \infty$, the right hand side of (3.7) decays to zero as $t \rightarrow \infty$. From this it is easy to see that $u(z(t))$ is a diverging curve in $N$. Fix a large $t_{0}$, and let $\gamma_{t}$ be the geodesic joining $u\left(z\left(t_{0}\right)\right)$ and $u(z(t))$. Passing to a subsequence $\gamma_{t}$ will converge to a ray in $N$. Let $\theta_{1}$ be the point at the ideal boundary of $N$ corresponding to this ray, then it is easy to see from Lemma 3.1 that $\lim _{t \rightarrow \infty} u(z(t))=\theta_{1}$.

Lemma 3.3. For $1 \leq i \leq m+2$, if $L$ is a horizontal ray with the same horizontal asymptotic direction as $H_{i}$, then $u(L)$ is asymptotic to $u\left(H_{i}\right)$. More precisely, if $L$ is given by $z(t), t \geq 0$, then $u(z(t)) \rightarrow \theta_{i}$, where $\theta_{i}$ is the point in the ideal boundary of $\mathbb{H}$ in Lemma 3.2.

Proof. If we parametrize $L$ and $H_{i}$ by the arclength parameter $t$, then $L(t)$ and $H_{i}(t)$ are connected by a vertical line $V_{t}(s), 0 \leq s \leq h$ for some 
fixed $h$, where $s$ is the arclength of $V_{t}$. By (3.6) the length $l_{t}$ of $u\left(V_{t}\right)$ is equal to $\int_{0}^{h} \sqrt{e-2} d s$. By Lemma $1.2, l_{t} \rightarrow 0$ as $t \rightarrow \infty$. Hence $\left.\operatorname{dist}_{N}\left(u(L(t)), u\left(H_{i}(t)\right)\right)\right) \rightarrow 0$ as $t \rightarrow \infty$. This completes the proof of the lemma.

Lemma 3.4. There is a positive function $\epsilon(R)$ with $\lim _{R \rightarrow \infty} \epsilon(R)=0$, such that if the distance of a horizontal trajectory $L$ from the zeros of $\Phi$ in the $\phi$-metric is $R$, then there are $\theta_{i}$ and $\theta_{j}$ in the ideal boundary of $N$, such that $\operatorname{dist}_{N}(u(L), \gamma) \leq \epsilon(R)$, where $\gamma$ is the geodesic joining $\theta_{i}$ and $\theta_{j}$. Here $\theta_{i}$ and $\theta_{j}$ are the points as in Lemma 3.2.

Proof. By Lemma 3.2 and 3.3, if $L$ is a horizontal trajectory near infinity in $\mathbb{C}$, and if we parametrized $L$ by $z(t),-\infty<t<\infty$, then there is $\theta_{i}$ and $\theta_{j}$ such that $\lim _{t \rightarrow \infty} u(z(t))=\theta_{i}$ and $\lim _{t \rightarrow \infty} u(z(t))=\theta_{j}$. As in the proof of Lemma 3.2, the result follows easily from Lemma 3.1

Lemma 3.5. Let $\theta_{1}, \ldots, \theta_{m+2}$ be the points in the ideal boundary of $N$ in Lemma 3.2, and let $P$ be the convex hull of the $\theta_{i}^{\prime} s$. Then $u(\mathbb{C}) \subset P$.

Proof. For any $z \in \mathbb{C}$, we can find a sequence of polygons $G_{k}$ containing $z$ such that the sides of $G_{k}$ consists of $m+2$ horizontal lines and $m+2$ vertical lines. We may choose $G_{k}$ so that each horizontal line is contained in a horizontal trajectory which is asymptotic to two consecutive directions, and so that the length of each vertical lines in the $\phi$-metric is bounded by a fixed constant. Moreover, the distance of the sides of $G_{k}$ from the zeroes of $\phi$ with respect to the $\phi$-metric tends to $\infty$ as $k \rightarrow \infty$. By Lemma 3.2-3.4, $\operatorname{dist}_{N}\left(P, u\left(\partial G_{k}\right)\right) \rightarrow 0$ as $k \rightarrow \infty$. By the maximum principle for harmonic maps, we see that $u(z) \in P$.

Theorem 3.6. Let $u$ be an orientation preserving harmonic diffeomorphism from $\mathbb{C}$ into a simply connected surface $N$ with Gaussian curvature bounded between two negative constants. Suppose $\|\partial u\|^{2}|d z|^{2}$ is a complete metric on $\mathbb{C}$ and the Hopf differential is $\phi d z^{2}$ where $\phi$ is a polynomial of degree $m$. Then there are exactly $m+2$ distinct points $\theta_{1}, \ldots, \theta_{m+2}$ in the ideal boundary of $N$ such that the image of $u$ is the convex hull of the set $\left\{\theta_{i}\right\}_{i=1}^{m+2}$. Moreover, the image of a horizontal trajectory of $\phi d z^{2}$ will be asymptotic to the geodesic joining two consecutive points of $\left\{\theta_{i}\right\}_{i=1}^{m+2}$. 
Proof. Let $\theta_{1}, \ldots, \theta_{m+2}$ be the points in Lemma 3.2. Suppose $\theta_{i}$ 's are not distinct, then by Gauss-Bonnet theorem, we have

$$
\int_{P}\left(-K_{N}\right)<m \pi
$$

where $K_{N}$ is the Gaussian curvature of $N$. By Lemma 3.4, and Theorem 1.3 .

$$
\begin{aligned}
m \pi & =\int_{u(\mathbb{C})}\left(-K_{N}\right) \\
& \leq \int_{P}\left(-K_{N}\right)<m \pi .
\end{aligned}
$$

This is a contradiction. Therefore, $\theta_{i}$ 's are distinct, and $u(\mathbb{C})=P$. The last statement follows from Lemma 3.2.

\section{References.}

[A] K. Akutagawa, Harmonic diffeomorphisms of the hyperbolic plane, to appear Trans. Amer. Math. Soc., 1990.

[ANT1] K. Akutagawa, S. Nishikawa and A. Tachikawa, Harmonic maps of unbounded convex polygons in the hyperbolic plane, in Geometry and its applications, Proceedings of the Workshop in honor of Morio Obata, Keio University, Nov. 1991, World Scientific, Singapore, 1993.

[ANT2] Harmonic maps between unbounded convex polyhedra in hyperbolic space, Invent. Math. 115(1994), 391-404.

[AN] S. Nishikawa and K. Akutagawa, The Gauss map and spacelike surfaces with prescribed mean curvature in Minkowski 3-Space, Tôhoku Math. J. 42(1990), 67-82.

[BF] C. Blanc, F. Fiala, Le type d'une surface et sa courbure totale, Comment. Math. Helv. 14(1941), 230-233.

[C] E. Calabi, Examples of Bernstein problems for some nonlinear equations, Proc. Symp. Pure and Appl. Math. of the American Math. Soc. 15(1968), 223-230.

[CY] S. Y. Cheng and S. T. Yau, Maximal spacelike hypersurfaces in the Lorentz-Minkowski spaces, Ann. Math. 104(1976), 407-419.

[CG] S. S. Chern and S. Goldberg, On the volume-decreasing property of a class of real harmonic mappings, Amer. J. Math. 97(1975), 133-147. 
112 Zheng-Chao Han, Luen-Fai Tam, Andrejs Treibergs and Tom Wan

[CT1] H. I. Choi and A. Treibergs, New examples of harmonic diffeomorphisms of the hyperbolic plane to itself, Manuscripta Math. 62(1988), 249-256.

[CT2] Gauss map of spacelike constant mean curvature hypersurface of Minkowski Space, J. Diff. Geom. 32(1990), 775-817.

[CT3] Hyperbolicity of constant mean curvature surfaces of Minkowski Space, Submitted to Proceedings of the first Pacific Rim Conference in Geometric Analysis held at The Chinese University of Hong Kong, Dec. 16-19, 1992., International Press

[F] R. Finn, On a class of conformal metrics, with application to differential geometry in the large, Comment. Math. Helv. 40(1965), 1-30.

[GT] D. Gilbarg and N. S. Trudinger, Elliptic Partial Differential Equations of Second Order, 2nd Edition, Springer-Verlag, 1983.

[Ha] Z. C. Han, Some Remarks Concerning the Geometric Behavior of Harmonic Maps between Surfaces, Preprint (1994).

[HN] J. Hano and K. Nomizu, Surfaces of revolution with constant mean curvature in Lorentz-Minkowski Space, Tôhoku Math. J. 36(1984), 427-437.

[He] E. Heinz, Über die Losungen der Minimalfächengleichung, Nach. Akad. Wiss. Göttingen Math. Phys. Kl. II 1952, 51-56.

[Ht] P. Hartman, On homotopic harmonic maps, Canadian J. Math. 19(1967), 673-687.

[Hu] A. Huber, On subharmonic functions and differential geometry in the large, Comment. Math. Helv. 32(1957), 13-72.

[I] T. Ishihara, The harmonic Gauss map in a generalized sense, J. London Math. Soc. 26(1982), 104-112.

[LT1] P. Li and L. F. Tam, The heat equation and harmonic maps of complete manifolds, Invent. Math. 105(1991), 1-46.

[LT2] Uniqueness and regularity of proper harmonic maps, Ann. Math. 137(1993), 167-201.

[LT3] Uniqueness and regularity of proper harmonic maps II, Indiana Math. J. 42(1993), 591-635.

[LTW] P. Li, L. F. Tam and J. P. Wang, Harmonic diffeomorphisms between Hadamard manifolds, to appear in Trans. AMS

[Mi] V. M. Miklyukov, Some characterization of the parabolicity and hyperbolicity by boundary sets of surfaces, preprint 1994. 
[M] T. K. Milnor, Harmonic maps and classical surface theory in Minkowski Space, Trans. Amer. Math Soc. 280(1983), 161-185.

[My] Y. Minsky, Harmonic maps, length, and energy in Teichmüller space, J. Diff. Geom. 35(1992), 151-217.

[O] R. Osserman, A Survey of Minimal Surfaces, Dover, 1986.

[S] R. Schoen, The role of harmonic mappings in rigidity and deformation problems, in Collection: Complex Geometry (Osaka, 1990), Lecture Notes in Pure and Applied Mathematics, Dekker, New York 143(1993), 179-200.

[SY] R. Schoen \& S. T. Yau, On Univalent Harmonic Maps between Surfaces, Invent. Math. 44(1978), 265-278.

[St] K. Strebel, Quadratic Differentials, Springer-Verlag, 1984.

[TW] L. F. Tam and T. Y.-H. Wan, Harmonic diffeomorphisms into CartanHadamard surfaces with prescribed Hopf differentials, to appear Comm. Anal. Geom.

[T1] A. Treibergs, Entire Spacelike Hypersurfaces of constant mean curvature in Minkowski Space, Invent. Math. 66(1982), 39-56.

[T2] Constant mean curvature hypersurfaces of Minkowski space and related problems, in Proceedings of the KAIST Mathematics Worksop in Analysis and Geometry, vol. 6, edotors D. B. Choi and J. W. Yim, Korean Advanced Institute for Science and Technology Mathematics Research Center, Taejon, Korea, 1991, 95-158.

[W] T. Wan, Constant mean curvature surface, harmonic maps, and univeral Teichmüller space, J. Diff. Geom. 35(1992), 643-657.

[WA] T. Wan and T. Au, Parabolic constant mean curvature spacelike surfaces, Proc. Amer. Math. Soc. 1992.

[Wo] M. Wolf, High energy degeneration of harmonic maps between surfaces and rays in Teichmüller space, Topology 30(1991), 517-540.

RUGTERS UNIVERSITY

New BRUNSWICK, NJ 08903

UNIVERSITY OF CALIFORNIA

IRVINE, CA 92717

UNIVERSITY OF UTAH

Salt Lake City, Utah 84109 
114 Zheng-Chao Han, Luen-Fai Tam, Andrejs Treibergs and Tom Wan AND

The Chinese University of Hong Kong HONG KONG

Received February 17Th, 1995. 